\title{
THE BURR TYPE XII DISTRIBUTION WITH SOME STATISTICAL PROPERTIES
}

\author{
Devendra Kumar ${ }^{1 *}$ \\ ${ }^{1}$ Department of Statistics Central University of Haryana, india
}

\begin{abstract}
This article addresses the various mathematical and statistical properties of the Burr type XII distribution (such as quantiles, moments, moment generating function, hazard rate, conditional moments, mean residual lifetime, mean past lifetime, mean deviation about mean and median, stochasic ordering, stress-strength parameter, various entropies, Bonferroni and Lorenz curves and order statistics) are derived. We discuss some exact expressions and recurrence relations for the single and product moments of upper record values. Further, using relations of single moments, we have tabulated the means and variances of upper record values from samples of sizes up to 10 for various values of the $\alpha$ and $\beta$. Finally a characterization of this distribution based on conditional moments of record values and recurrence relation of kth record values is presented.
\end{abstract}

Key words: Upper record values, single and product moments, recurrence relations, characterization, Burr type XII distribution.

\section{Introduction}

Record values are used in many statistical applications, statistical modeling and inference involving data pertaining to weather, athletic events, economics, life testing studies and so on. For example, Guinness World Records, fastest time taken to recite the periodic table of the elements or shortest ever tennis matches both in terms of number of games and duration of time or fastest in- door marathon, etc. People make several attempts to make records but records are made only when the attempt is a success. Usually, we don't get the data on all of the attempts made to break the records around the world. The data that we have are the records. Because of importance of record values in many fields of application, these kind of ordered data have been extensively studied in the literature. There are hundreds of papers and several books published on record-breaking data and its distributional properties (see, for instance, Chandler (1952), Resnick (1973), Shorrock (1973), Glick (1978), Nevzorov (1987), Ahsan- ullah (1995), Balakrishnan and Ahsanullah (1993, 1994), Grunzien and Szynal (1997) and Arnold et al. (1992, 1998)). Hence, it is pertinent that one has to study the properties based on records. The concept of recurrence relations has its own importance. They are useful in reducing the number of

\footnotetext{
*Corresponding author. Email: devendrastats@gmail.com
} 
operations neces- sary to obtain a general form for the function under consideration. Furthermore, they are used in characterizing the distributions, which is an important area, permitting the identification of population distribution from the properties of the sample. Recurrence relations and identities have attained importance as it reduces the amount of direct computation, time and labour.

The Burr type XII distribution was first introduced in the literature by Burr (1942) and has gained special attention in the last two decades or so due to its broad applications in different fields including the area of reliability, failure time modeling and acceptance sampling plan and so on. For instance, Wang and Keats (1996) used the maximum likelihood method for obtaining point and in- terval estimators of the parameters of the Burr XII distribution. Abdel-Ghaly et al. (1997) applied the Burr type XII distribution to measure software reliability. Zimmer et al. (1998) also studied statistical and probabilistic properties of the Burr type XII distribution and described its relationship to other distributions used in reliability analyses. Moore and Papadopoulos (2000) derived Bayesian estimators of the parameter and the reliability function for the Burr type XII distribution under three different loss functions. Ali Mousa and Jaheen (2002) considered Bayesian estimation of the parameters of the Burr distribution based on progressively censored samples. Wu and $\mathrm{Yu}$ (2005) proposed pivotal quantities to test the shape parameter and establish confidence interval of the shape param- eter of the Burr type XII distribution under the failurecensored plan. Li et al. (2007) proposed the empirical estimators of reliability performances for Burr XII distribution under LINEX loss function. Wu et al. (2007) used the maximum likelihood method to derive the point estimators of the parameters for Burr type XII distribution. Prediction of future observables from Burr type XII distribu- tion was studied by AL-Hussaini (2003), ALHussaini and Ahmad (2003), Kumar (2016) have derived ratio and inverse moments of MarshallOlkin extended Burr type XII distribution based on lower generalized order statistics and Constant partially accelerated life tests for Burr XII distribution with progressive type II censoring was investigated by Abdel-Hamid (2009) and Singh and Shukla (2017), among others.

The two parameter Burr type XII distribution which is denoted by Burr XII $(\alpha, \beta)$ has the following probability density function (pdf ) of the form

$$
f(x ; \alpha, \beta)=\alpha \beta x^{\alpha-1}\left(1+x^{\alpha}\right)^{-(\beta+1)}, \quad x>0, \quad \alpha, \beta>0
$$

and a cumulative distribution function (cdf)

$$
F(x ; \alpha, \beta)=1-\left(1+x^{\alpha}\right)^{-\beta}, x>0, \quad \alpha, \beta>p,
$$

where $\alpha$ and $\beta$ are shape and scale parameters, respectively. Note that the Figure 1 illustrate some of the possible shapes of the pdf of the Burr XII $(\alpha, \beta)$ for selected values of the parameters $\alpha$ and $\beta$. 

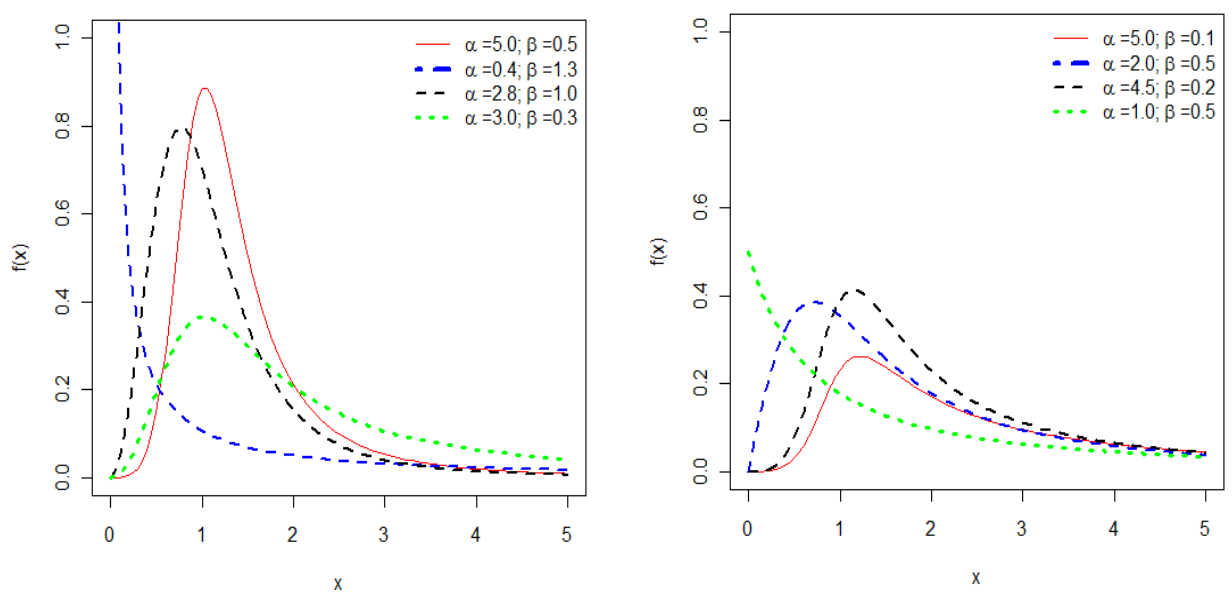

Figure 1: $\operatorname{Burr} \operatorname{XII}(\alpha, \beta)$ density function

Recurrence relations for single and product moments of kth record values from Weibull, Pareto, generalized Pareto, Burr, exponential and Gumble distribution are derived by Pawalas and Szynal (1998, 1999, 2000). Sultan (2007), Kumar(2015, 2016) and Kumar et al. (2015, 2017) have established recurrence relations for moments of kth record values for modified Weibull, generalized Rayleigh, type-I generalized half logistic and Kumaraswamy-Burr III distributions respectively.

The rest of this paper proceeds as follows. In Section 2, we first describe briefly the preliminaries of record values. We derive some mathematical and statical properties of this distribution in Section 3. In Section 4, we derive the explicit expressions and recurrence relations for single and product moments. In Section 5, characterization of the distribution based on conditional moments of record values and recurrence relation of kth record values are established. Numerical results are presented in Section 6. Finally, in Section 7, we make some concluding remarks.

\section{Record Values and Preliminaries}

Let $\left\{\mathrm{X}_{\mathrm{n}}, \mathrm{n} \geq 1\right\}$ be a sequence of identically independently distributed (i.i.d) random variables with pdf $f(x)$ and cumulative distribution function (cdf) $F(x)$. The $j$-th order statistics of a sample $\left(X_{1}, X_{2}, \ldots, X_{n}\right)$ is denoted by $X j: n$. For a fix $k \geq 1$ we define the sequence $\{U$ $(\mathrm{k})(\mathrm{n}), \mathrm{n} \geq 1\}$ of $\mathrm{k}$ upper record times of $\mathrm{X}_{1}, \mathrm{X}_{1}, \ldots$ as follows:

$\mathrm{U}^{(\mathrm{k})}(1)=1$,

$\mathrm{U}^{(\mathrm{k})}(\mathrm{n}+1)=\min \left\{\mathrm{j}>\mathrm{U}^{(\mathrm{k})}(\mathrm{n}): \mathrm{X}_{\mathrm{j}: \mathrm{j}+\mathrm{k}+1}>\mathrm{X}_{\mathrm{U}}(\mathrm{k})_{(\mathrm{n})}: \mathrm{U}^{(\mathrm{k})}(\mathrm{n})+\mathrm{k} \quad 1\right\}$ 
The sequences $\left\{\mathrm{Z}_{n}^{(k)}, \mathrm{n} \geq 1\right\}$ with $\mathrm{Z}_{n}^{(k)}=\mathrm{X}_{\mathrm{k}: \mathrm{U}(\mathrm{k})(\mathrm{n})+\mathrm{k}-1}, \mathrm{n}=1,2, \ldots$, are called the sequences of kth upper record values of $\left\{X_{n}, n \geq 1\right\}$. For convenience, we shall also take $\mathrm{Z}_{0}^{(k)}=0$. Note that $\mathrm{k}=1$ we have $\mathrm{Y}_{n}^{(1)}=\mathrm{X}_{\mathrm{U}(\mathrm{n})}, \mathrm{n} \geq 1$, i.e. record values of $\left\{\mathrm{X}_{\mathrm{n}}, \mathrm{n} \geq 1\right\}$.

The joint pdf of kth upper record values $\mathrm{Z}_{1}^{(k)}, \mathrm{Z}_{2}^{(k)}, \ldots, \mathrm{Z}_{n}^{(k)}$ can be given as the joint pdf of kth upper record values of $\left\{-X_{n}, n \geq 1\right\}$, Pawlas and Szynal (1998)

$$
f_{z_{1}^{(k)}, \ldots, z_{n}^{(k)}}\left(z_{1}, \ldots, z_{n}\right)=k^{n}\left(\prod_{i=1}^{n-1} \frac{f\left(z_{i}\right)}{1-F\left(z_{i}\right)}\right)\left[1-F\left(z_{n}\right)\right]^{k-1} f\left(z_{n}\right), z_{1}<z_{2}<\ldots<z_{n} .
$$

In view of above equation, the marginal pdf of $\mathrm{X}_{U(n)}^{(k)}, \mathrm{n} \geq 1$ is given by

$$
f_{X_{n}^{(k)}}(x)=\frac{k^{n}}{(n-1) !}[-\ln (\bar{F}(x))]^{n-1}[\bar{F}(x)]^{k-1} f(x)
$$

and the joint pdf of $\mathrm{X}_{m)}^{(k)}$ and $\mathrm{X}_{n}^{(k)}, 1 \leq \mathrm{m}<\mathrm{n}, \mathrm{n}>2$ is given by

$$
\begin{aligned}
& f_{X_{m}^{(k)}, X_{n}^{(k)}}(x, y)=\frac{k^{n}}{(m-1) !(n-m-1) !}[-\ln \mid(\bar{F}(x))]^{m-1} \\
& \quad \times[-\ln \bar{F}(y)+\ln \bar{F}(x)]^{n-m-1}[\bar{F}(y)]^{k-1} \frac{f(x)}{\bar{F}(x)} f(y), x<y,
\end{aligned}
$$

where $\overline{\mathrm{F}}(\mathrm{x})=1-\mathrm{F}(\mathrm{x})$.

\section{Statistical and Mathematical properties}

In this section, we give some important statistical and mathematical measures for the Burr $\operatorname{XII}(\alpha, \beta)$ like quantiles, moment generating function, moments, hazard rate and mean residual life functions, mean past lifetime, conditional moments, Stochastic ordering, mean deviation about mean and median, Shannon and R'enyi entropy, order statistics, stress strength parameter.

Quantile functions are in widely used in general statistics and often find repre-sentations in terms of lookup tables for key percentiles.From(2), it follows that the $\mathrm{p}$-th quantile function $\mathrm{F}^{-1}(\mathrm{p})$ of the Burr $\mathrm{XII}(\alpha, \beta)$ is given by

$$
Q(p)=\left[(1-p)^{-1 / \beta}-1\right]^{1 / \alpha}, \quad 0<p<1 .
$$

In particular, the first three quantiles, Q1, Q2 and Q3, can be obtained by setting $\mathrm{p}=0.25, \mathrm{p}$ $=0.5$ and $\mathrm{p}=0.75$ in equation (5).

The effects of the shape parameters $\alpha$ and $\beta$ on the skewness and kurtosis can be considered based on quantile measures. The Bowley skewness (Kenney \& Keeping 1962) is one of the earliest skewness measures defined by 


$$
B=\frac{Q\left(\frac{3}{4}\right)+Q\left(\frac{1}{4}\right)-2 Q\left(\frac{1}{4}\right)}{Q\left(\frac{3}{4}\right)-Q\left(\frac{1}{4}\right)},
$$

where $\left.\mathrm{Q}(3 / 4)=\left((0.25)^{-1 / \beta}-1\right)^{1 / \alpha}, \mathrm{Q}(1 / 4)=\left((0.75)^{-1 / \beta}-1\right)\right)^{1 / \alpha}$ and $\mathrm{Q}(1 / 2)=\left((0.50)^{-1 / \beta}-1\right)^{1 / \alpha}$.

Since only the middle two quartiles are considered and the outer two quartiles are ignored, this adds robustness to the measure. The Moors kurtosis (Moors 1988) is defined as

$$
M=\frac{Q\left(\frac{3}{8}\right)-Q\left(\frac{1}{8}\right)+Q\left(\frac{7}{8}\right)-Q\left(\frac{5}{8}\right)}{Q\left(\frac{6}{8}\right)-Q\left(\frac{2}{8}\right)},
$$

where $\mathrm{Q}(3 / 8)=\left((0.625)^{-1 / \beta}-1\right)^{1 / \alpha}, \mathrm{Q}(1 / 8)=\left((0.875)^{-1 / \beta}-1\right)^{1 / \alpha}, \mathrm{Q}(7 / 8)=\left((0.125)^{-1 / \beta}-1\right)^{1 / \alpha}$ and $\mathrm{Q}(5 / 8)=\left((0.375)^{-1 / \beta}-1\right)^{1 / \alpha}$.

Clearly, $\mathrm{M}>0$ and there is good concordance with the classical kurtosis measures for some distributions. These measures are less sensitive to outliers and they exist even for distributions without moments. For the standard normal distribution, these measures are 0 (Bowley) and 1.2331 (Moors).

We hardly need to emphasize the necessity and importance of the moments in any statistical analysis especially in applied work. Some of the most important features and characteristics of a distribution can be studied through moments (e.g., tendency, dispersion, skewness, and kurtosis). If the random variable $X$ having the $\operatorname{Burr} \operatorname{XII}(\alpha, \beta)$. It is easy to obtain the kth moment of $X$ as the following form

$$
E\left(X^{k}\right)=\int_{0}^{\infty} x^{k} f(x) d x=\alpha \beta \sum_{p=0}^{\infty} \frac{(-1)^{p} \Gamma\left(\frac{k}{\alpha}+1\right)}{p ! \Gamma\left(\frac{k}{\alpha}+1-p\right)[\alpha(\beta+p)-k]} .
$$

Hence in particular we have

$$
\begin{gathered}
E(X)=\int_{0}^{\infty} x f(x) d x=\alpha \beta \sum_{p=0}^{\infty} \frac{(-1)^{p} \Gamma\left(\frac{1}{\alpha}+1\right)}{p ! \Gamma\left(\frac{1}{\alpha}+1-p\right)[\alpha(\beta+p)-1]}, \\
E\left(X^{2}\right)=\int_{0}^{\infty} x^{2} f(x) d x=\alpha \beta \sum_{p=0}^{\infty} \frac{(-1)^{p} \Gamma\left(\frac{2}{\alpha}+1\right)}{p ! \Gamma\left(\frac{2}{\alpha}+1-p\right)[\alpha(\beta+p)-2]}, \\
E\left(X^{3}\right)=\int_{0}^{\infty} x^{3} f(x) d x=\alpha \beta \sum_{p=0}^{\infty} \frac{(-1)^{p} \Gamma\left(\frac{3}{\alpha}+1\right)}{p ! \Gamma\left(\frac{3}{\alpha}+1-p\right)[\alpha(\beta+p)-3]}
\end{gathered}
$$

and

$$
E\left(X^{4}\right)=\int_{0}^{\infty} x^{4} f(x) d x=\alpha \beta \sum_{p=0}^{\infty} \frac{(-1)^{p} \Gamma\left(\frac{2}{\alpha}+1\right)}{p ! \Gamma\left(\frac{4}{\alpha}+1-p\right)[\alpha(\beta+p)-4]} .
$$

The variance, skewness and kurtosis measures can now be calculated using the relations

$$
\begin{gathered}
\operatorname{Var}(X)=E\left(X^{2}\right)-E^{2}(X), \\
\text { Skewness }(X)=\frac{E\left(X^{3}\right)-3 E(X) E\left(X^{2}\right)+2 E^{3}(X)}{\operatorname{Var}^{3 / 2}(X)},
\end{gathered}
$$




$$
\operatorname{Kurtosis}(X)=\frac{E\left(X^{4}\right)-4 E(X) E\left(X^{3}\right)+6 E\left(X^{2}\right) E^{2}(X)-3 E^{4}(X)}{\operatorname{Var}^{2}(X)} .
$$

\subsection{Moment Generating Function}

Many of the interesting characteristics and features of a distribution can be obtained via its moment generating function and moments. Let $\mathrm{X}$ denote a random variable with the probability density function (1). By definition of moment generating function of $\mathrm{X}$ and using (1), we have

$$
\begin{aligned}
M_{X}(t) & =E\left(e^{t x}\right)=\int_{0}^{\infty} e^{t x} f(x) d x \\
& =\alpha \beta \sum_{i=0}^{\infty} \sum_{p=0}^{\infty} \frac{(-1)^{p} t^{i} \Gamma\left(\frac{i}{\alpha}+1\right)}{i ! p ! \Gamma\left(\frac{i}{\alpha}+1\right)[\alpha(\beta+p)-i]} .
\end{aligned}
$$

\subsection{Hazard Function and Survival (reliability) function}

The basic tools for studying the ageing and reliability characteristics of the system are the hazard rate (HR) and the mean residual lifetime (MRL). The HR and the

Table 1 Mean,variance,skewness,kurtosis and coefficient of variation for $\beta=5$ and some values of $\alpha$

\begin{tabular}{cccccc}
\hline$\alpha$ & Mean & Variance & Skewness & Kurtosis & CV \\
\hline 1 & 0.814695 & 0.625704 & 0.007627 & 3.712924 & 0.970933 \\
2 & 0.599551 & 0.455234 & 0.000202 & 0.876736 & 1.125359 \\
3 & 0.520236 & 0.402862 & 0.031172 & 0.839334 & 1.220050 \\
4 & 0.477495 & 0.371550 & 0.09062 & 0.899750 & 1.276556 \\
5 & 0.450534 & 0.349823 & 0.149939 & 0.971513 & 1.312794 \\
6 & 0.431915 & 0.333685 & 0.201877 & 1.038074 & 1.337427 \\
7 & 0.418265 & 0.321175 & 0.245632 & 1.096193 & 1.354938 \\
8 & 0.407820 & 0.311178 & 0.282115 & 1.145926 & 1.367842 \\
9 & 0.399567 & 0.302999 & 0.312509 & 1.188334 & 1.377625 \\
10 & 0.392879 & 0.296180 & 0.337962 & 1.224531 & 1.385221 \\
\hline
\end{tabular}

It appears from the results that the mean of $\operatorname{Burr} \operatorname{XII}(\alpha, \beta)$ is greater than its variance.

MRL deal with the residual lifetime of the system. The HR gives the rate of failure of the system immediately after time $\mathrm{x}$, and the MRL measures the expected value of the remaining lifetime of the system, provided that it has survived up to time $\mathrm{x}$. Thus the hazard rate function of the $\operatorname{Burr} \operatorname{XII}(\alpha, \beta)$ distribution is given by

$$
h(x)=\frac{f(x)}{1-F(x)}=\frac{\alpha \beta x^{\alpha-1}\left(1+x^{\alpha}\right)^{-(\beta+1)}}{1-\left(1+x^{\alpha}\right)^{-\beta}}, x>0, \quad \alpha, \beta>0
$$

The reliability function $\mathrm{R}(\mathrm{x})$, which is the probability of an item not failing prior to some time $t$, is defined by $R(x)=1-F(x)$. The reliability function of a $\operatorname{Burr} \operatorname{XII}(\alpha, \beta)$ is given by 


$$
R(x)=\left(1+x^{\alpha}\right)^{-\beta} .
$$

\subsection{Conditional moments}

For the Burr $\mathrm{XII}(\alpha, \beta)$ distribution, it can be easily seen that the conditional moments, $\mathrm{E}\left(\mathrm{X}^{\mathrm{n}} \mid \mathrm{X}\right.$ $>\mathrm{x})$, can be written as $\mathrm{E}\left(\mathrm{X}^{\mathrm{n}} \mid \mathrm{X}>\mathrm{x}\right)=\frac{1}{S(x)} \mathrm{J}_{\mathrm{n}}(\mathrm{x})$, where

$$
J_{n}(x)=\int_{x}^{\infty} y^{n} f(y) d y=\alpha \beta \sum_{p=0}^{\infty} \frac{(-1)^{p} \Gamma\left(\frac{k}{\alpha}+1\right)[\bar{F}(x)]^{\frac{p}{\beta}-\frac{k}{\alpha \beta}}}{p ! \Gamma\left(\frac{k}{\alpha}+1-p\right)[\alpha(p+\beta)-k]},
$$

where $\Gamma()=.\int_{0}^{\infty} t^{\mathrm{a}-1} \mathrm{e}^{-\mathrm{t}} \mathrm{dt}$ represents the complete gamma function and $\mathrm{S}(\mathrm{x})=1-\mathrm{F}(\mathrm{x})$ defined in (9).
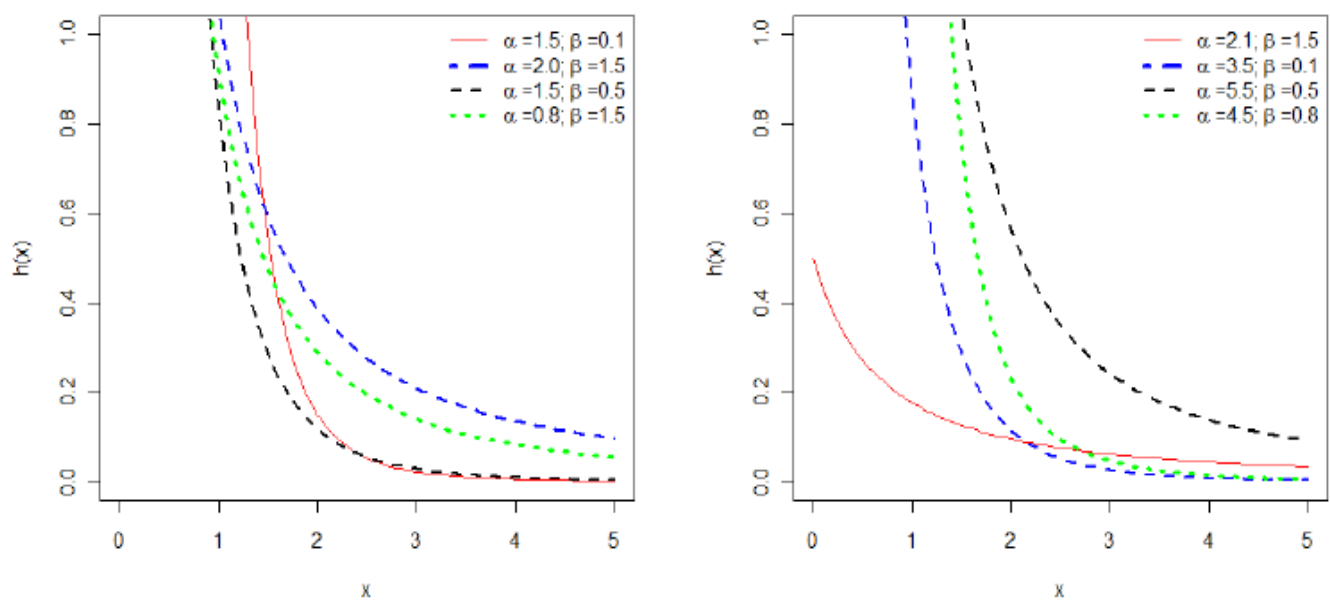

Figure 2: $\operatorname{Burr} \operatorname{XII}(\alpha, \beta)$ hazard function

An application of the conditional moments is the mean residual life (MRL). MRL function is the expected remaining life, $\mathrm{X}-\mathrm{x}$, given that the item has survived to time $\mathrm{x}$. Thus, in life testing situations, the expected additional lifetime given that a component has survived until time $\mathrm{x}$ is called the (MRL). The MRL function in terms of the first conditional moment as

$$
m_{X}(x)=E(X-x \mid X>x)=\frac{1}{S(x)} J_{1}(x)-x .
$$

where $\mathrm{J}_{1}(\mathrm{x})$ can be obtained from (10) where $\mathrm{n}=1$.

Another application of the conditional moments is the mean deviations about the mean and the median. They are used to measure the dispersion and the spread in a population from the center. If we denote the median by $\mathrm{M}$, then the mean deviations about the mean and the median can be calculated as 


$$
\delta_{\mu}=\int_{0}^{\infty}|x-\mu| f(x) d x=2 \mu F(\mu)-2 \mu+2 J_{1}(\mu)
$$

and

$$
\delta_{M}=\int_{0}^{\infty}|x-M| f(x) d x=2 J_{1}(M)-\mu,
$$

respectively. Where $\mathrm{J}_{1}(\mu)$ and $\mathrm{J}_{1}(\mathrm{M})$ can obtained from (10). Also, $F(\mu)$ and $F(M)$ are easily calculated from (2).

\subsection{Mean Past Lifetime (MPL)}

In a real life situation, where systems are usually not monitored continuously, we often might be interested in knowing the history of the system e.g. when the individual components have failed. Let us assume that a component with lifetime $\mathrm{X}$ has failed at or some time before $\mathrm{X}$, $\mathrm{x} \geq 0$. Therefore the conditional random variable $\mathrm{x}-\mathrm{X} \mid \mathrm{X} \leq \mathrm{x}$ will state the time elapsed from the failure of the component given that its lifetime is less than or equal to $\mathrm{x}$. Hence, the mean past lifetime (MPL) of the component can be defined as

$$
\begin{aligned}
k(x) & =E[x-X \mid X \leq x]=x-\frac{\int_{0}^{x} F(t) d t}{F(x)} \\
& =x-\frac{\alpha \beta}{\left[1-\left(1+x^{\alpha}\right)^{-\beta}\right]} \sum_{p=0}^{\infty} \frac{(-1)^{p} \Gamma\left(\frac{1}{\alpha}+1\right)}{p ! \Gamma\left(\frac{1}{\alpha}+1-p\right)[\alpha(p+\beta)-1]} \\
& \times\left[\left\{1-\left(1+x^{\alpha}\right)^{-\beta}\right\}^{\frac{1}{\alpha}-p-\beta}-1\right] .
\end{aligned}
$$

\subsection{Stochastic ordering}

Stochastic ordering is a tool used to study structural properties of complex stochastic systems. For example, it is useful for controlling congestion in information transfer over the Internet, for deening treatment-related trends in clustered binary data, and ordering expected welfare income under differentent mechanisms of allocating rewards. There are different types of stochastic orderings which are useful in ordering random variables in terms of different properties. Here we consider four different stochastic orders, namely, the usual, the hazard rate, the mean residual life, and the likelihood ratio order for two independent $\operatorname{Burr} \operatorname{XII}(\alpha, \beta)$ random variables under a restricted parameter space. If $\mathrm{X}$ and $\mathrm{Y}$ are independent random variables with CDFs FX and FY respectively, then $\mathrm{X}$ is said to be smaller than $\mathrm{Y}$ in the

- $\quad$ stochastic order $\left(X \leq_{\mathrm{st}}(\mathrm{Y})\right)$ if $\mathrm{F}_{\mathrm{X}}(\mathrm{x}) \geq \mathrm{F}_{\mathrm{Y}}(\mathrm{x})$ for all $\mathrm{x}$

- hazard rate order $\left(X \leq_{h r}(Y)\right)$ if $h_{X}(x) \geq h_{Y}(x)$ for all $x$

- mean residual life order $\left(\mathrm{X} \leq_{\mathrm{mrl}}(\mathrm{Y})\right)$ if $\mathrm{m}_{\mathrm{X}}(\mathrm{x}) \geq \mathrm{m}_{\mathrm{Y}}(\mathrm{x})$ for all $\mathrm{x}$

- likelihood ratio order $\left(X \leq_{\operatorname{lr}}(Y)\right)$ if $f_{X}(x) / f_{y}(x)$ decrease in $x$. 
The following results due to Shaked and Shanthikumar (1994) are well known for establishing stochastic ordering of distributions.

$$
\begin{aligned}
X \leq_{l r} Y \Rightarrow X \quad & \leq_{h r} \quad Y \Rightarrow X \leq_{m l r} Y \\
& \Downarrow \\
& X \leq_{s t} Y
\end{aligned}
$$

The $\operatorname{Burr} \operatorname{XII}(\alpha, \beta)$ distribution is ordered with respect to the strongest "likelihood ratio" ordering as shown in the following theorem. It shows the flexibility of two parameter $\operatorname{Burr} \operatorname{XII}(\alpha$, B) distribution.

Theorem 1. Let $X \sim \operatorname{BurrXII}\left(\alpha_{1}, \beta_{1}\right)$ and $Y \sim \operatorname{BurrXII}\left(\alpha_{2}, \beta_{2}\right)$.If $\alpha_{1}=\alpha_{1}=\alpha$ and $\beta_{1} \geq \beta_{2}$, then $\mathrm{X} \leq_{\mathrm{lr}} \mathrm{Y}, \mathrm{X} \leq_{\mathrm{hr}} \mathrm{Y}, \mathrm{X} \leq_{\mathrm{mrl}} \mathrm{Y}$ and $\mathrm{X} \leq_{\mathrm{st}} \mathrm{Y}$. Proof. The likelihood ratio is

$$
\frac{f_{X}(x)}{f_{Y}(x)}=\frac{\alpha_{1} \beta_{1} x^{\alpha_{1}-1}\left(1+x^{\alpha_{1}}\right)^{-\left(\beta_{1}-1\right)}}{\alpha_{2} \beta_{2} x^{\alpha_{2}-1}\left(1+x^{\alpha_{2}}\right)^{-\left(\beta_{2}-1\right)}}
$$

Thus,

$$
\frac{d}{d x} \log \frac{f_{X}(x)}{f_{Y}(x)}=\left(\alpha_{1}-\alpha_{2}\right)-\frac{\left(\beta_{1}+1\right) x^{\alpha_{1}} \log x}{1+x^{\alpha_{1}}}+\frac{\left(\beta_{2}+1\right) x^{\alpha_{2}} \log x}{1+x^{\alpha_{2}}} .
$$

Now if $\alpha_{1}=\alpha_{2}=\alpha$ and $\beta_{1} \geq \beta_{2}$ then $\frac{\mathrm{d}}{\mathrm{dx}} \log \frac{f x(x)}{f y(y)} \leq 0$, which implies that $\mathrm{X} \leq_{\mathrm{lr}} \mathrm{Y}$ and hence $\mathrm{X} \leq_{\mathrm{lr}} \mathrm{Y}, \mathrm{X} \leq_{\mathrm{hr}} \mathrm{Y}, \mathrm{X} \leq_{\mathrm{mrl}} \mathrm{Y}$ and $\mathrm{X} \leq_{\mathrm{st}} \mathrm{Y}$.

\subsection{Bonferroni and Lorenz curve}

Boneferroni and Lorenz curves are proposed by Boneferroni (1930). These curves have applications not only in economics to study income and poverty, but also in other fields like reliability, demography, insurance and medicine. They are define as

$$
B(p)=\frac{1}{p \mu} \int_{0}^{q} x f(x) d x
$$

and

$$
L(p)=\frac{1}{\mu} \int_{0}^{q} x f(x) d x
$$

respectively, where $\mu=E(X)$ and $q=F^{-1}(p)$. By using (1), one can reduce (17) and (18) to

$$
\begin{aligned}
B(p) & =\frac{\alpha \beta}{p \mu} \sum_{k=0}^{\infty} \frac{(-1)^{k} \Gamma\left(\frac{1}{\alpha}+1\right)}{k ! \Gamma\left(\frac{1}{\alpha}+1-k\right)[\alpha(k+\beta)-1]} \\
& \times\left[\left\{1-\left(1+q^{\alpha}\right)^{-\beta}\right\}^{\frac{1}{\alpha}-k-\beta}-1\right],
\end{aligned}
$$




$$
\begin{aligned}
L(p) & =\frac{\alpha \beta}{\mu} \sum_{k=0}^{\infty} \frac{(-1)^{k} \Gamma\left(\frac{1}{\alpha}+1\right)}{k ! \Gamma\left(\frac{1}{\alpha}+1-k\right)[\alpha(k+\beta)-1]} \\
& \times\left[\left\{1-\left(1+q^{\alpha}\right)^{-\beta}\right\}^{\frac{1}{\alpha}-k-\beta}-1\right],
\end{aligned}
$$

respectively.

\subsection{Entropies}

An entropy provides an excellent tool to quantify the amount of information (or uncertainty) contained in a random observation regarding its parent distribution (population). A large value of entropy implies the greater uncertainty in the data. The concept of entropy is important in different areas such as physics, probability and statistics, communication theory, economics, etc. Several measures of entropy have been studied and compared in the literature. If $\mathrm{X}$ has the probability distribution function $f(\cdot)$ Renyi entropy (Renyi, 1961) can be expressed as

$$
\begin{aligned}
H_{\delta}(x) & =\frac{1}{1-\delta} \ln \left(\int_{0}^{\infty} f^{\delta}(x) d x\right), \quad \delta>0, \quad \delta \neq 1 \\
& =\frac{1}{1-\delta}[(\rho-1) \ln \alpha+\rho \ln \beta]+\frac{1}{1-\delta} \\
& \times \ln \left[\frac{\Gamma\left(\frac{\rho(\alpha-1)+1}{\alpha}\right) \Gamma\left(2+\rho(\beta+1)-\frac{\rho(\alpha-1)+1}{\alpha}\right)}{\Gamma(\rho(\beta+1)+2)}\right],
\end{aligned}
$$

when $\delta \rightarrow 1$, the Renyi entropy converges to the Shannon entropy.For furthers details, see Song (2001).

\subsection{Stress Strength Reliability}

In reliability theory, the stress-strength model describes the life of a component or item which has a random strength $\mathrm{X} 1$ that is subjected to a random stress $\mathrm{X}_{2}$ : The component fails instantaneously when the stress applied to it surpass the strength, and the component will function satisfactorily/acceptably whenever $\mathrm{X}_{1}>\mathrm{X}_{2}$ : So, $\mathrm{R}=\operatorname{Pr}\left(\mathrm{X}_{1}>\mathrm{X}_{2}\right)$ is a measure of component reliability. Its applicability is found in many spheres especially in engineering such as structures, deterioration of rocket motors, fatigue failure of aircraft structures, the aging of concrete pressure vessels, etc. Extensive work on estimation of reliability of stress-strength models has been done for the well known standard distributions. However, there are still some distributions (including generalizations of the well- known distributions) for which the form of $\mathrm{R}$ has not been investigated. Here, we derive the reliability $\mathrm{R}$ when $\mathrm{X}_{1}$ and $\mathrm{X}_{2}$ are independent random variables distributed with parameters $(\alpha 1, \beta 1)$ and $(\alpha 2, \beta 2)$, then 


$$
\begin{aligned}
R & =P\left(X_{2}<X_{1}\right)=\int_{0}^{\infty} f_{1}(x) F_{2}(x) d x \\
& =\alpha_{1} \beta_{1} \int_{0}^{\infty} \frac{x^{\alpha_{1}-1}}{\left(1+x^{\alpha_{1}}\right)^{\beta_{1}+1}}\left(1-\frac{1}{\left(1+x^{\alpha_{2}}\right)^{\beta_{2}}}\right) d x .
\end{aligned}
$$

If $\alpha_{1}=\alpha_{2}=\alpha$ then

$$
R=\beta_{1}\left(\frac{\Gamma\left(1+\beta_{1}-\alpha\right)}{\Gamma\left(1+\beta_{1}\right)}-\frac{\Gamma\left(1+\beta_{1}+\beta_{2}-\alpha\right)}{\Gamma\left(1+\beta_{1}+\beta_{2}\right)}\right) .
$$

\subsection{Order Statistics}

Moments of order statistics play an important role in quality control testing and reliability to predict the failure of future items based on the times of few early failures. Thus, the kth order statistic of a sample is its kth smallest value. For a sample of size $n$, the nth order statistic (or largest order statistic) is the maximum, that is,

$$
\mathrm{X}_{(\mathrm{n})}=\max \left\{\mathrm{X}_{1}, \mathrm{X}_{2}, \ldots, \mathrm{X}_{\mathrm{n}}\right\}
$$

The sample range is the difference between the maximum and minimum. It is clearly a function of the order statistics:

$$
\text { range }\left\{X_{1}, X_{2}, \ldots, X_{n}\right\}=X_{(n)}-X_{(1)} \text {. }
$$

We know that if $X_{(1)} \leq \cdots \leq X_{(n)}$ denotes the order statistic of a random sample $X_{1}, \ldots$, $X_{n}$ from a continuous population with cdf $G_{X}(x)$ and pdf $g_{X}(x)$ then the pdf of $X_{(j)}$ is given by

$$
g_{X_{(j)}}(x)=\frac{n !}{(j-1) !(n-j) !} g_{X}(x)\left(G_{X}(x)\right)^{j-1}\left(1-G_{X}(x)\right)^{n-j},
$$

for $\mathrm{j}=1, \ldots, \mathrm{n}$. The pdf and cdf of the $\mathrm{jth}$ order statistic for a $\operatorname{Burr} \operatorname{XII}(\alpha, \beta)$ distribution is given by

$$
g_{X_{(j)}}(x)=\frac{\alpha \beta x^{\alpha-1} n !}{(j-1) !(n-j) !} \sum_{u=0}^{j-1}(-1)^{u}\left(\begin{array}{c}
j-1 \\
u
\end{array}\right)\left(1+x^{\alpha}\right)^{-\beta(n-j+u+1)-1}
$$

and

$$
G_{j: n}(x)=\sum_{l=j}^{n} \sum_{u=0}^{l}(-1)^{u}\left(\begin{array}{c}
n \\
l
\end{array}\right)\left(\begin{array}{l}
l \\
u
\end{array}\right)\left[\left(1+x^{\alpha}\right)^{-\beta}\right]^{u+n-l} .
$$

The kth moments of $\mathrm{Xj}: \mathrm{n}$ can be expressed 


$$
\begin{aligned}
E\left[X_{j: n}^{k}\right] & =\frac{\alpha \beta n !}{(j-1) !(n-j) !} \sum_{p=0}^{\infty} \sum_{u=0}^{j-1}(-1)^{u+p}\left(\begin{array}{c}
j-1 \\
u
\end{array}\right) \\
& \times \frac{\Gamma\left(\frac{k}{\alpha}+1\right)}{p ! \Gamma\left(\frac{k}{\alpha}+1-p\right)[\alpha\{\beta(u+n-j+1)+p\}-k]} .
\end{aligned}
$$

\subsection{Distribution of Minimum, Maximum and Median}

Let $X_{1}, X_{2}, \ldots, X n$ be independently identically distributed order random vari- ables from the Burr $X I I(\alpha, \beta)$ having first, last and median order probability density functions are given by the following

$$
\begin{aligned}
g_{1: n}(x) & =n\left[1-G\left(x_{(1)}, \Phi\right)\right]^{n-1} g\left(x_{(1)}, \Phi\right) \\
& =\alpha \beta n x^{\alpha-1}\left(1+x^{\alpha}\right)^{-(\beta n+1)}, \\
g_{n: n}(x) & =n\left[G\left(x_{(n)}, \Phi\right)\right]^{n-1} g\left(x_{(n)}, \Phi\right) \\
& =\alpha \beta n x^{\alpha-1}\left(1+x^{\alpha}\right)^{-(\beta+1)}\left[1-\left(1+x^{\alpha}\right)\right]^{n-1},
\end{aligned}
$$

and

$$
\begin{aligned}
g_{m+1: n}(\tilde{x}) & =\frac{(2 m+1) !}{m ! m !}[G(x, \Phi)]^{m}[1-G(x, \Phi)]^{m} g\left(x_{(n)}, \Phi\right) \\
& =\frac{\alpha \beta(2 m+1) !}{m ! m !} x^{\alpha-1}\left[1-\left(1+x^{\alpha}\right)^{-\beta}\right]^{m}\left(1+x^{\alpha}\right)^{-\beta(m+1)-1},
\end{aligned}
$$

where $\Phi=(\alpha, \beta)$

\subsection{Joint distribution of the ith and jth order Statistics}

The joint distribution of the the ith and jth order statistics from $\operatorname{Burr} \operatorname{XII}(\alpha, \beta)$ is

$$
\begin{aligned}
g_{i, j: n}\left(x_{i}, x_{j}\right) & =\frac{n !}{(i-1) !(j-i-1) !(j-1) !}\left[G\left(x_{i}, \Phi\right)\right]^{i-1}\left[G\left(x_{j}, \Phi\right)-G\left(x_{i}, \Phi\right)\right]^{j-1-1} \\
& \times\left[1-G\left(x_{i}, \Phi\right)\right]^{n-j} g\left(x_{i}, \Phi\right) g\left(x_{j}, \Phi\right) \\
& =\frac{n ! \alpha^{2} \beta^{2} x_{i}^{\alpha-1} x_{j}^{\alpha-1}}{(i-1) !(j-i-1) !(j-1) !}\left[1-\left(1+x_{i}^{\alpha}\right)^{-\beta}\right]^{i-1}\left(1+x_{i}^{\alpha}\right)^{-(\beta+1)} \\
& \times\left[\left(1+x_{i}^{\alpha}\right)^{-\beta}-\left(1+x_{j}^{\alpha}\right)^{-\beta}\right]^{j-i-1}\left(1+x_{j}^{\alpha}\right)^{-\beta(n-j+1)-1} .
\end{aligned}
$$

If $\mathrm{i}=1$ and $\mathrm{j}=\mathrm{n}$ we get the joint distribution of the minimum and maximum of order statistics 


$$
\begin{aligned}
g_{1, n: n}\left(x_{1}, x_{n}\right) & =n(n-1)\left[G\left(x_{n}, \Phi\right)-G\left(x_{1}, \Phi\right)\right]^{n-2} g\left(x_{1}, \Phi\right) g\left(x_{n}, \Phi\right) \\
& =\alpha^{2} \beta^{2} n(n-1)\left[\left(1+x_{1}^{\alpha}\right)^{-\beta}-\left(1+x_{n}^{\alpha}\right)^{-\beta}\right]^{n-2} \\
& \times\left(1+x_{1}^{\alpha}\right)^{-(\beta+1)}\left(1+x_{n}^{\alpha}\right)^{-(\beta+1)} x_{1}^{\alpha-1} x_{n}^{\alpha-1} .
\end{aligned}
$$

\section{Moments of record values}

In this section we derive explicit expressions and recurrence relations for single and product moments of kth upper record values from $\operatorname{Burr} \operatorname{XII}(\alpha, \beta)$.

\subsection{Relations for Single moments}

We shall first establish explicit expressions for single moments of kth upper record values, $\mathrm{E}\left(\left(X_{U(n)}^{(k)}\right)^{\mathrm{r}}\right)=\mu_{n: k}^{(r)}$. Theorem 1 gives an explicit expression for single moments of kth upper record values and $r=1,2, \ldots$. Theorem 2 gives an explicit expression for single moments of kth upper record values and $\mathrm{r}$ a negative integer.

Theorem 1. For the $\operatorname{Burr} \operatorname{XII}(\alpha, \beta)$ given in (1) with fixed parameters $\alpha, \beta>0, k, n=1,2, \ldots$, and $\mathrm{r}=1,2, \ldots$, we have

$$
\mu_{n: k}^{(r)}=(\beta k)^{n} \sum_{p=0}^{r / \alpha}(-1)^{p} \frac{\Gamma\left(\frac{r}{\alpha}+1\right)}{p ! \Gamma\left(\frac{r}{\alpha}+1-p\right)\left(\beta k-\frac{r}{\alpha}+p\right)^{n}},
$$

where $\Gamma($.$) denotes the complete gamma function defined by \Gamma(\mathrm{a})=, \int_{0}^{\infty} t^{a-1} \mathrm{e}^{\mathrm{t} d t}$.

Proof. Using (3), we have

$$
\begin{aligned}
\mu_{n: k}^{(r)} & =\frac{k^{n}}{(n-1) !} \int_{0}^{\infty} x^{r}[-\ln (\bar{F}(x))]^{n-1}[\bar{F}(x)]^{k-1} f(x) d x \\
& =\frac{(\beta k)^{n}}{(n-1) !} \int_{0}^{1}(1-t)^{\frac{r}{\alpha}} t^{\beta k-\frac{r}{\alpha}-1}[-\ln t]^{n-1} d t \\
& =\frac{(\beta k)^{n}}{(n-1) !} \sum_{p=0}^{r / \alpha}(-1)^{p} \frac{\Gamma\left(\frac{r}{\alpha}+1\right)}{p ! \Gamma\left(\frac{r}{\alpha}+1-p\right)} \int_{0}^{1} t^{\beta k+p-\frac{r}{\alpha}-1}[-\ln t]^{n-1} d t \\
& =\frac{(\beta k)^{n}}{(n-1) !} \sum_{p=0}^{r / \alpha}(-1)^{p} \frac{\Gamma\left(\frac{r}{\alpha}+1\right)}{p ! \Gamma\left(\frac{r}{\alpha}+1-p\right)} \int_{0}^{\infty} w^{n-1} e^{-\left(\beta k+p-\frac{r}{\alpha}\right) w} d w
\end{aligned}
$$

where $w=-\ln t$ and $t=[\bar{F}(x)]^{1 / \beta}$. The result follows from the definition of the complete gamma function.

In particular, the mean kth record values and the variance kth record values ar 


$$
\mu_{n: k}^{(1)}=(\beta k)^{n} \sum_{p=0}^{1 / \alpha}(-1)^{p} \frac{\Gamma\left(\frac{1}{\alpha}+1\right)}{p ! \Gamma\left(\frac{1}{\alpha}+1-p\right)\left(\beta k-\frac{1}{\alpha}+p\right)^{n}},
$$

and

$$
\begin{aligned}
\sigma_{n: k}^{2} & =\mu_{n: k}^{(2)}-\left[\mu_{n: k}\right]^{2} \\
& =(\beta k)^{n} \sum_{p=0}^{2 / \alpha}(-1)^{p} \frac{\Gamma\left(\frac{2}{\alpha}+1\right)}{p ! \Gamma\left(\frac{2}{\alpha}+1-p\right)\left(\beta k-\frac{2}{\alpha}+p\right)^{n}}-\left[\mu_{n: k}\right]^{2},
\end{aligned}
$$

respectively. If $\mathrm{k}=1$ these reduce to the corresponding upper record values for the $\operatorname{Burr} \operatorname{XII}(\alpha, \beta)$.

Theorem 2. For the Burr XII $(\alpha, \beta)$ given in (1) with fixed parameters $\alpha, \beta>0$,

$\mathrm{k}, \mathrm{n}=1,2, \ldots$, and $\mathrm{r}$ a negative integer, we have

$$
\mu_{n: k}^{(r)}=(\beta k)^{n} \sum_{p=0}^{\infty}(-1)^{p} \frac{\Gamma\left(\frac{r}{\alpha}+1\right)}{p ! \Gamma\left(\frac{r}{\alpha}+1-p\right)\left(\beta k-\frac{r}{\alpha}+p\right)^{n}} .
$$

Proof. Similar to the proof of Theorem 1.

Theorem 3 establishes a recurrence relation for $\mu_{n: k}^{(r)}$. This result holds for positive as well as negative $r$.

Theorem 3: Under assumptions of Theorem 1, and convention $\mu_{n: k}^{(r)}=1, \mu_{0: k}^{(r)}=0$, we have,

$$
\mu_{n: k}^{(r)}=\mu_{n-1: k}^{(r)}+\frac{r}{\alpha \beta k}\left[\mu_{n: k}^{(r-\alpha)}+\mu_{n: k}^{(r)}\right] .
$$

Proof. Clearly, from (1) and (2), we see that

$$
\alpha \beta \bar{F}(x)=x\left(1+x^{-\alpha}\right) f(x)=x f(x)+x^{-\alpha} f(x) .
$$

Therefore, from (3) we have

$$
\mu_{n: k}^{(r)}=\frac{k^{n}}{(n-1) !} \int_{0}^{\infty} x^{r}[-\ln (\bar{F}(x))]^{n-1}[\bar{F}(x)]^{k-1} f(x) d x .
$$

Integrating by parts of (38), we obtain

$$
\begin{aligned}
\mu_{n: k}^{(r)} & =\mu_{n-1: k}^{(r)}+\frac{r k^{n}}{k(n-1) !} \int_{0}^{\infty} x^{r-1}[-\ln (\bar{F}(x))]^{n-1}[\bar{F}(x)]^{k} d x \\
& =\mu_{n-1: k}^{(r)}+\frac{r k^{n}}{k(n-1) !} \int_{0}^{\infty} x^{r-1}[-\ln (\bar{F}(x))]^{n-1}[\bar{F}(x)]^{k-1}\left(\frac{x+x^{-\alpha+1}}{\alpha \beta}\right) f(x) d x \\
& =\mu_{n-1: k}^{(r)}+\frac{r k^{n}}{\alpha \beta k(n-1) !} \int_{0}^{\infty} x^{r}[-\ln (\bar{F}(x))]^{n-1}[\bar{F}(x)]^{k-1} f(x) d x \\
& +\frac{r k^{n}}{\alpha \beta k(n-1) !} \int_{0}^{\infty} x^{r-\alpha}[-\ln (\bar{F}(x))]^{n-1}[\bar{F}(x)]^{k-1} f(x) d x
\end{aligned}
$$

The result follows. 
In particular, upon setting $\mathrm{n}=1$ in Theorem 3 , we deduce the following result. Corollary 1. For the $\operatorname{Burr} X I I(\alpha, \beta)$ given in (1),

$$
\mu_{1: k}^{(r)}=\frac{r}{\alpha \beta k}\left[\mu_{1: k}^{(r-\alpha)}+\mu_{1: k}^{(r)}\right] .
$$

If $\mathrm{k}=1$ these reduce to the corresponding upper record values for the $\operatorname{Burr} \operatorname{XII}(\alpha, \beta)$.

\subsection{Relations for Product moments}

We shall first establish explicit expressions for the product moment of kth upper record values, $\mathrm{E}\left(X_{U(m, n): k}^{(r, s)}\right)=\mu_{m, n, k}^{(r, s)}$ Theorem 4 gives an explicit expression for $1 \leq \mathrm{m}<\mathrm{n}$ and $\mathrm{r}$, $\mathrm{s}=1,2, \ldots$. Theorem 5 gives an explicit expression for $1 \leq \mathrm{m}<\mathrm{n}, \mathrm{r}=1,2, \ldots$ and $\mathrm{s}$ a negative integer. Theorem 6 gives an explicit expression for $1 \leq \mathrm{m}<\mathrm{n}, \mathrm{s}=1,2, \ldots$ and $\mathrm{r}$ a negative integer. Theorem 7 gives an explicit expression for $1 \leq \mathrm{m}<\mathrm{n}$ and both $\mathrm{r}$ and $\mathrm{s}$ negative integers.

Theorem 4. For the Burr $\operatorname{XII}(\alpha, \beta)$ given in (1) and for $1 \leq \mathrm{m}<\mathrm{n}$ and $\mathrm{r}, \mathrm{s}=1,2, \ldots$,

$$
\begin{aligned}
\mu_{m, n: k}^{(r, s)} & =(\beta k)^{n} \sum_{p=0}^{s / \alpha} \sum_{q=0}^{r / \alpha}(-1)^{p+q} \frac{\Gamma\left(\frac{s}{\alpha}+1\right)}{p ! q ! \Gamma\left(\frac{s}{\alpha}+1-p\right) \Gamma\left(\frac{r}{\alpha}+1-q\right)} . \\
& \times \frac{\Gamma\left(\frac{r}{\alpha}+1\right)}{\left(\beta k+p-\frac{s}{\alpha}\right)^{n-m}\left(\beta k+p+q-\frac{r+s}{\alpha}\right)^{m}} .
\end{aligned}
$$

Proof. Using (4), we hav 


$$
\begin{aligned}
& \mu_{m, n: k}^{(r, s)}=\frac{k^{n}}{(m-1) !(n-m-1) !} \int_{0}^{\infty} \int_{x}^{\infty} x^{r} y^{s}[-\ln (\bar{F}(x))]^{m-1} \frac{f(x)}{[\bar{F}(x)]} \\
& \times \quad[-\ln (\bar{F}(y))+\ln (\bar{F}(x))]^{n-m-1}[\bar{F}(x)]^{k-1} f(y) d y d x \\
& =\frac{k^{n}}{(m-1) !(n-m-1) !} \int_{0}^{\infty} x^{r}[-\ln (\bar{F}(x))]^{m-1} \frac{f(x)}{[\bar{F}(x)]} \\
& \times\left(\int_{x}^{\infty} y^{s}[-\ln (\bar{F}(y))+\ln (\bar{F}(x))]^{n-m-1}[\bar{F}(x)]^{k-1} f(y) d y\right) d x \\
& =\frac{k^{n}}{(m-1) !(n-m-1) !} \int_{0}^{\infty} x^{r}[-\ln (\bar{F}(x))]^{m-1} \frac{f(x)}{[\bar{F}(x)]} \\
& \times\left(\int_{0}^{\infty}\left[\frac{1-\left\{e^{-w} \bar{F}(x)\right\}^{1 / \beta}}{\left\{e^{-w} \bar{F}(x)\right\}^{1 / \beta}}\right]^{s / \alpha} w^{n-m-1}\left[e^{-w} \bar{F}(x)\right]^{k} d w\right) d x \\
& =\frac{k^{n}}{(m-1) !} \sum_{p=0}^{\infty}(-1)^{p} \frac{\Gamma\left(\frac{s}{\alpha}+1\right)}{p ! \Gamma\left(\frac{s}{\alpha}+1-p\right)\left(k+\frac{p}{\beta}-\frac{s}{\alpha \beta}\right)^{n-m}} \\
& \times \quad \int_{0}^{\infty} x^{r}[-\ln (\bar{F}(x))]^{m-1}[\bar{F}(x)]^{k+\frac{p}{\beta}-\frac{s}{\alpha \beta}-1} f(x) d x \\
& =\frac{k^{n}}{(m-1) !} \sum_{p=0}^{s / \alpha}(-1)^{p} \frac{\Gamma\left(\frac{s}{\alpha}+1\right)}{p ! \Gamma\left(\frac{s}{\alpha}+1-p\right)\left(k+\frac{p}{\beta}-\frac{s}{\alpha \beta}\right)^{n-m}} \\
& \times \quad \int_{0}^{1}(1-z)^{r / \alpha}[-\ln z]^{m-1} z^{\beta k-p-\left(\frac{s+r}{\alpha}\right)-1} d z \\
& =\frac{k^{n}}{(m-1) !} \sum_{p=0}^{s / \alpha}(-1)^{p} \frac{\Gamma\left(\frac{s}{\alpha}+1\right)}{p ! \Gamma\left(\frac{s}{\alpha}+1-p\right)\left(k+\frac{p}{\beta}-\frac{s}{\alpha \beta}\right)^{n-m}} \\
& \times \beta^{m} \sum_{q=0}^{r / \alpha}(-1)^{q} \frac{\Gamma\left(\frac{r}{\alpha}+1\right)}{q ! \Gamma\left(\frac{r}{\alpha}+1-q\right)} \int_{0}^{\infty} e^{-\left(\beta k+p+q-\left\{\frac{r+s}{\alpha}\right\}\right) t} t^{m-1} d t,
\end{aligned}
$$

where $\mathrm{w}=-\ln (\overline{\mathrm{F}}(\mathrm{y}))-\ln (\overline{\mathrm{F}}(\mathrm{x})), \mathrm{z}=[\overline{\mathrm{F}}(\mathrm{x})]^{1 / \beta}$ and $\mathrm{t}=-\ln \mathrm{z}$. The result follows by using complete gamma function to calculate the integral in (41).

The proof is complete.

As a check, put $\mathrm{s}=0$ in (40) and use (33), we have $\mu_{m, n,}^{(r, 0)}=\mu_{n}^{(r)}$.

For simplicity, we denote the $(1,1)$ - th moment of $U_{n}$ and $U_{m}$, which are also called the simple product moment of these records, by $\mu_{\mathrm{U}(\mathrm{m}, \mathrm{n})}$. The simple product moments are used for evaluating the covariances, in other words

$$
\sigma_{m, n}=\operatorname{cov}\left(U_{m}, U_{n}\right)=\mu_{m, n}-\mu_{m} \mu_{n}
$$


Theorem 5. For the Burr $\operatorname{XII}(\alpha, \beta)$ given in (1) and for $1 \leq \mathrm{m}<\mathrm{n}$ and $\mathrm{r}=1,2, \ldots$, and and $\mathrm{s}$ a negative integer,

$$
\begin{aligned}
\mu_{m, n: k}^{(r, s)} & =(\beta k)^{n} \sum_{p=0}^{\infty} \sum_{q=0}^{r / \alpha}(-1)^{p+q} \frac{\Gamma\left(\frac{s}{\alpha}+1\right)}{p ! q ! \Gamma\left(\frac{s}{\alpha}+1-p\right) \Gamma\left(\frac{r}{\alpha}+1-q\right)} \\
& \times \frac{\Gamma\left(\frac{r}{\alpha}+1\right)}{\left(\beta k+p-\frac{s}{\alpha}\right)^{n-m}\left(\beta k+p+q-\frac{r+s}{\alpha}\right)^{m}} .
\end{aligned}
$$

Proof. Similar to the proof of Theorem 4 .

Theorem 6. For the Burr $X I I(\alpha, \beta)$ given in (1) and for $1 \leq m<n$ and $s=1$, $2, \ldots$, and and $\mathrm{r}$ a negative integer,

$$
\begin{aligned}
\mu_{m, n: k}^{(r, s)} & =(\beta k)^{n} \sum_{p=0}^{s / \alpha} \sum_{q=0}^{\infty}(-1)^{p+q} \frac{\Gamma\left(\frac{s}{\alpha}+1\right)}{p ! q ! \Gamma\left(\frac{s}{\alpha}+1-p\right) \Gamma\left(\frac{r}{\alpha}+1-q\right)} \\
& \times \frac{\Gamma\left(\frac{r}{\alpha}+1\right)}{\left(\beta k+p-\frac{s}{\alpha}\right)^{n-m}\left(\beta k+p+q-\frac{r+s}{\alpha}\right)^{m}} .
\end{aligned}
$$

Proof. Similar to the proof of Theorem 4 .

Theorem 7. For the Burr $\operatorname{XII}(\alpha, \beta)$ given in (1) and for $1 \leq \mathrm{m}<\mathrm{n}$ and and both $\mathrm{r}$ and s negative integers,

$$
\begin{aligned}
\mu_{m, n: k}^{(r, s)} & =(\beta k)^{n} \sum_{p=0}^{\infty} \sum_{q=0}^{\infty}(-1)^{p+q} \frac{\Gamma\left(\frac{s}{\alpha}+1\right)}{p ! q ! \Gamma\left(\frac{s}{\alpha}+1-p\right) \Gamma\left(\frac{r}{\alpha}+1-q\right)} \\
& \times \frac{\Gamma\left(\frac{r}{\alpha}+1\right)}{\left(\beta k+p-\frac{s}{\alpha}\right)^{n-m}\left(\beta k+p+q-\frac{r+s}{\alpha}\right)^{m}} .
\end{aligned}
$$

Proof. Similar to the proof of Theorem 4.

Theorem 8 establishes a recurrence relation for $\mu_{m, n: k}^{(r, s)}$. This result holds for positive as well as negative values of $r$ and $s$.

Theorem 8.For the Burr $\operatorname{XII}(\alpha, \beta)$ given in (1) and $1 \leq \mathrm{m} \leq \mathrm{n}-2$,

$$
\mu_{m, n: k}^{(r, s)}=\mu_{m, n-1: k}^{(r, s)}+\frac{s}{\alpha \beta k}\left[\mu_{m, n: k}^{(r, s-\alpha)}+\mu_{m, n: k}^{(r, s)}\right] .
$$

Proof: From equation (4) for $1 \leq \mathrm{m} \leq \mathrm{n}-2$,

$$
\begin{aligned}
\mu_{m, n: k}^{(r, s)} & =\frac{k^{n}}{(m-1) !(n-m-1) !} \int_{0}^{\infty} \int_{x}^{\infty} x^{r} y^{s}[-\ln (\bar{F}(x))]^{m-1} \frac{f(x)}{[\bar{F}(x)]} \\
& \times[-\ln (\bar{F}(y))+\ln (\bar{F}(x))]^{n-m-1}[\bar{F}(y)]^{k-1} f(y) d y d x .
\end{aligned}
$$

By integrating by parts with respect to $y$, we obtain 


$$
\begin{aligned}
\mu_{m, n: k}^{(r, s)} & =\mu_{m, n-1: k}^{(r, s)}+\frac{s k^{n}}{k(m-1) !(n-m-1) !} \int_{0}^{\infty} \int_{x}^{\infty} x^{r} y^{s-1}[-\ln (\bar{F}(x))]^{m-1} \frac{f(x)}{[\bar{F}(x)]} \\
& \times[-\ln (\bar{F}(y))+\ln (\bar{F}(x))]^{n-m-1}[\bar{F}(y)]^{k} d y d x . \\
& =\mu_{m, n-1: k}^{(r, s)}+\frac{s k^{n}}{k(m-1) !(n-m-1) !} \int_{0}^{\infty} \int_{x}^{\infty} x^{r} y^{s-1}[-\ln (\bar{F}(x))]^{m-1} \frac{f(x)}{[\bar{F}(x)]} \\
& \times[-\ln (\bar{F}(y))+\ln (\bar{F}(x))]^{n-m-1}[\bar{F}(y)]^{k-1}\left(\frac{x+x^{-\alpha+1}}{\alpha \beta}\right) f(y) d y d x . \\
& =\mu_{m, n-1: k}^{(r, s)}+\frac{s k^{n}}{\alpha \beta k(m-1) !(n-m-1) !} \int_{0}^{\infty} \int_{x}^{\infty} x^{r} y^{s}[-\ln (\bar{F}(x))]^{m-1} \frac{f(x)}{[\bar{F}(x)]} \\
& \times[-\ln (\bar{F}(y))+\ln (\bar{F}(x))]^{n-m-1}[\bar{F}(y)]^{k-1} f(y) d y d x \\
& +\frac{s k^{n}}{\alpha \beta k(m-1) !(n-m-1) !} \int_{0}^{\infty} \int_{x}^{\infty} x^{r} y^{s-\alpha}[-\ln (\bar{F}(x))]^{m-1} \frac{f(x)}{[\bar{F}(x)]} \\
& \times[-\ln (\bar{F}(y))+\ln (\bar{F}(x))]^{n-m-1}[\bar{F}(y)]^{k-1} f(y) d y d x .
\end{aligned}
$$

The result follows.

In particular, upon setting $\mathrm{n}=\mathrm{m}+1$ in Theorem 8 , we deduce the following result.

Corollary 2. For the Burr $\operatorname{XII}(\alpha, \beta)$ given in (1) and $1 \leq \mathrm{m} \leq \mathrm{n}-2$,

$$
\mu_{m, m+1: k}^{(r, s)}=\mu_{m, m: k}^{(r, s)}+\frac{s}{\alpha \beta k}\left[\mu_{m, m+1: k}^{(r, s-\alpha)}+\mu_{m, m+1: k}^{(r, s)}\right] .
$$

\section{Characterization}

In this section, we characterize the $\operatorname{Burr} \operatorname{XII}(\alpha, \beta)$ based on conditional moments of upper record values and recurrence relation of single moments of kth upper record values.

Let $\mathrm{L}(\mathrm{a}, \mathrm{b})$ stand for the space of all integrable functions on $(\mathrm{a}, \mathrm{b})$. A sequence $(\mathrm{hn}) \subset \mathrm{L}(\mathrm{a}$, b) is called complete on $\mathrm{L}(\mathrm{a}, \mathrm{b})$ if for all functions $\mathrm{g} \in \mathrm{L}(\mathrm{a}, \mathrm{b})$ the condition

$$
\int_{a}^{b} g(x) f_{n}(x) d x=0, \quad n \in N
$$

implies $\mathrm{g}(\mathrm{x})=0$ a.e. on (a, b). We start with the following result of Lin (1986).

Proposition 1. Let $n_{0}$ be any fixed non-negative integer, $-\infty \leq a<b \leq \infty$ and $g(x) \geq 0$ an absolutely continuous function with $g^{\prime}(x) \neq 0$ a.e. on $(a, b)$. Then the sequence of functions $\left\{(g(x))^{n} e^{-g(x)}, \quad n \geq n_{0}\right\}$ is complete in $L(a, b)$ iff $g(x)$ is strictly monotone on $(a$, b).

Using the above Proposition we get a stronger version of Theorem 9.

Let $\{X n, n \geq 1\}$ be a sequence of i.i.d continuous random variables with $d f F$ $(x)$ and $\operatorname{pdf} f(x)$. Let $X_{U(n)}$ be the $n$-th upper record values, then the conditional $p d f$ of $X U(n)$ given $X_{U(m)}=x, 1 \leq m<n$ in view of (3) and (4), is 


$$
f\left(X_{U(n)} \mid X_{U(m)}=x\right)=\frac{1}{(n-m-1) !}[-\ln \bar{F}(y)+\ln \bar{F}(x)]^{n-m-1} \frac{f(y)}{\bar{F}(x)}, \quad x>y .
$$

Theorem 5: Let $X$ be an absolutely continuous random variable with df $F(x)$ and pdf $\mathrm{f}(\mathrm{x})$ on the support $(0, \infty)$, then for $m<n$,

$$
E\left[X_{U(n)} \mid X_{U(m)}=x\right]=\sum_{p=0}^{\infty} \frac{(-1)^{p+\frac{1}{\alpha}} \Gamma\left(\frac{1}{\alpha}+1\right)\left(1+x^{\alpha}\right)^{p / \beta}}{p ! \Gamma\left(\frac{1}{\alpha}+1-p\right)\left(1-\frac{p}{\beta}\right)^{n-m}} .
$$

if and only if

$$
F(x ; \alpha, \beta)=1-\left(1+x^{\alpha}\right)^{-\beta}, x>0, \alpha, \beta>0 .
$$

Proof: From (47), we have

$$
\begin{aligned}
E\left[X_{U(n)} \mid X_{U(m)}=x\right] & =\frac{1}{(n-m-1) !} \int_{x}^{\infty} y\left[\ln \left(\frac{\bar{F}(x)}{\bar{F}(y)}\right)\right]^{n-m-1} \frac{f(y)}{\bar{F}(x)} d y \\
& =\frac{1}{(n-m-1) !} \sum_{p=0}^{\infty} \frac{(-1)^{p+\frac{1}{\alpha}} \Gamma\left(\frac{1}{\alpha}+1\right)\left(1+x^{\alpha}\right)^{p / \beta}}{p ! \Gamma\left(\frac{1}{\alpha}+1-p\right)} \\
& \times \int_{0}^{\infty} t^{n-m+p-1} e^{-\left(1-\frac{p}{\beta}\right) t} d t
\end{aligned}
$$

where $\mathrm{t}=\ln \left(\frac{\overline{F(x)}}{F(y)}\right)$. The result follows by using the complete gamma function to calculate the integral in (49). The proof is complete.

To prove sufficient part, we have from (47) and (48)

$$
\frac{1}{(n-m-1) !} \int_{x}^{\infty} y[-\ln \bar{F}(y)+\ln \bar{F}(x)]^{n-m-1} f(y) d y=\bar{F}(x) H_{r}(x),
$$

where

$$
H_{r}(x)=\sum_{p=0}^{\infty} \frac{(-1)^{p+\frac{1}{\alpha}} \Gamma\left(\frac{1}{\alpha}+1\right)\left(1+x^{\alpha}\right)^{p / \beta}}{p ! \Gamma\left(\frac{1}{\alpha}+1-p\right)\left(1-\frac{p}{\beta}\right)^{n-m}} .
$$

Differentiating (50) both sides with respect to $\mathrm{x}$, we get

$$
-\frac{1}{(n-m-2) !} \int_{x}^{\infty} y[-\ln \bar{F}(y)+\ln \bar{F}(x)]^{n-m-2} \frac{f(x)}{\bar{F}(x)} f(y) d y=-f(x) H_{r}(x)+\bar{F}(x) H_{r}^{\prime}(x)
$$

or

$$
\frac{f(x ; \alpha, \lambda)}{\bar{F}(x ; \alpha, \lambda)}=\frac{H_{r}^{\prime}(x)}{\left[H_{r+1}(x)-H_{r}(x)\right]}=\frac{\alpha \beta}{x\left(1+x^{-\alpha-1}\right)},
$$

which proves that

$$
F(x ; \alpha, \beta)=1-\left(1+x^{\alpha}\right)^{-\beta}, x>0, \alpha, \beta>0 .
$$

Theorem 6. Let $\mathrm{X}$ be a non-negative random variable having an absolutely continuous distribution function $\mathrm{F}(\mathrm{x})$ with $\mathrm{F}(0)=0$ and $\mathrm{F}(\mathrm{x})>0$ for all $\mathrm{X}>0$. The 


$$
\mu_{n: k}^{(r)}=\mu_{n-1: k}^{(r)}+\frac{r}{\alpha \beta k}\left[\mu_{n: k}^{(r-\alpha)}+\mu_{n: k}^{(r)}\right] .
$$

holds for fixed $\alpha, \beta>0$, positive integer $\mathrm{k}$ and all positive integer $\mathrm{n}$ if and only if

$$
F(x ; \alpha, \beta)=1-\left(1+x^{\alpha}\right)^{-\beta}, x>0, \alpha, \beta>0 .
$$

Proof. The necessary part follows immediately from equation (37). On the other hand if the recurrence relation in equation (51) is satisfied, then on using equation (2), we have

$$
\begin{aligned}
& \frac{k^{n}}{(n-1) !} \int_{0}^{\infty} x^{r}[-\ln (\bar{F}(x))]^{n-1}[\bar{F}(x)]^{k-1} f(x) d x \\
= & \frac{k^{n}}{(n-2) !} \int_{0}^{\infty} x^{r}[-\ln (\bar{F}(x))]^{n-2}[\bar{F}(x)]^{k-1} f(x) d x \\
+ & \frac{r k^{n}}{\alpha \beta k(n-1) !} \int_{0}^{\infty} x^{r}[-\ln (\bar{F}(x))]^{n-1}[\bar{F}(x)]^{k-1} f(x) d x \\
+ & \frac{r k^{n}}{\alpha \beta k(n-1) !} \int_{0}^{\infty} x^{r-\alpha}[-\ln (\bar{F}(x))]^{n-1}[\bar{F}(x)]^{k-1} f(x) d x .
\end{aligned}
$$

Integrating the first integral on the right hand side of equation (52), by parts we get and simplifying the resulting expression, we find that

$$
\frac{r k^{n}}{k(n-1) !} \int_{0}^{\infty} x^{r-1}[-\ln (\bar{F}(x))]^{n-1}[\bar{F}(x)]^{k-1} d x\left\{\bar{F}(x)-\left(\frac{x}{\alpha \beta}+\frac{x^{-\alpha}}{\alpha \beta}\right) f(x)\right\}=0 .
$$

It now follows from Proposition 1

$$
\bar{F}(x)=\left(\frac{x}{\alpha \beta}+\frac{x^{-\alpha}}{\alpha \beta}\right) f(x),
$$

which proves that

$$
F(x, a, \beta)=1-\left(1+x^{\alpha}\right)^{-\beta}, x>0, a, \beta>0
$$

\section{Numerical Results}

The recurrence relations obtained in the preceding sections allow us to evaluate the means, variances and covariances of all order statistics for all sample sizes in a simple recursive manner. Means, variances and covariances of all order statistics can be used for various inferential purposes; for example, they are useful in determining BLUEs of location/scale parameters and best linear unbiased predictors (BLUPs) of censored failure times. More details on BLUEs and BLUPs based on order statistics can be seen in Balakrishnan and Cohen (1991) and Arnold et al. (1992).

In Table 1-2, we have computed the values of means for $n=1,2, \cdots 10$ and $\beta=0.5(0.5) 3.5$. One can see that the means are decreasing with respect to $n$ but increasing with respect to $\beta$. In Table 3-4, we have computed the variances and covariances for different values $\mathrm{m}$ and $\mathrm{n}$ and for 
different values of $\alpha$ and $\lambda$. We can see that variances are decreases for both $\alpha$ and $\lambda$ values increases.

Tables for the skewness, kurtosis, product moments and covariances of record values are not presented here but are available from the author on request. All computations here were performed using Mathematica. Mathematica like other algebraic manipulation packages allows for arbitrary precision, so the accuracy of the given values is not an issue.

Table 1. Means of record statistics for $\alpha=5$.

\begin{tabular}{|c|c|c|c|c|c|c|c|}
\hline$n$ & $\beta=0.5$ & $\beta=1.0$ & $\beta=1.5$ & $\beta=2.0$ & $\beta=2.5$ & $\beta=3.0$ & $\beta=3.5$ \\
\hline 1 & 0.403713 & 0.67897 & 0.87816 & 1.02739 & 1.14182 & 1.23102 & 1.30142 \\
\hline 2 & 0.400294 & 0.63927 & 0.77701 & 0.85292 & 0.89047 & 0.90386 & 0.90190 \\
\hline 3 & 0.371889 & 0.55633 & 0.63296 & 0.65103 & 0.63829 & 0.61004 & 0.57474 \\
\hline 4 & 0.340351 & 0.47271 & 0.50165 & 0.48275 & 0.44415 & 0.39954 & 0.35533 \\
\hline 5 & 0.310098 & 0.39770 & 0.39258 & 0.35298 & 0.30454 & 0.25775 & 0.21634 \\
\hline 6 & 0.282122 & 0.33304 & 0.30514 & 0.25605 & 0.20703 & 0.16480 & 0.13052 \\
\hline 7 & 0.256542 & 0.27825 & 0.23625 & 0.18483 & 0.13997 & 0.10476 & 0.07827 \\
\hline 8 & 0.233242 & 0.23219 & 0.18248 & 0.13299 & 0.09429 & 0.06633 & 0.04674 \\
\hline 9 & 0.212045 & 0.19363 & 0.14074 & 0.09549 & 0.06335 & 0.04187 & 0.02783 \\
\hline 10 & 0.19277 & 0.16143 & 0.10845 & 0.06846 & 0.04248 & 0.02638 & 0.01653 \\
\hline \multicolumn{8}{|c|}{ Table 2. Means of record statistics for $\alpha=10$} \\
\hline$n$ & $\beta=0.5$ & $\beta=1.0$ & $\beta=1.5$ & $\beta=2.0$ & $\beta=2.5$ & $\beta=3.0$ & $\beta=3.5$ \\
\hline 1 & 0.448409 & 0.82174 & 1.14275 & 1.42353 & 1.67183 & 1.89322 & 2.09188 \\
\hline 2 & 0.446642 & 0.79519 & 1.06729 & 1.28084 & 1.44893 & 1.58124 & 1.68506 \\
\hline 3 & 0.429914 & 0.73826 & 0.95424 & 1.10238 & 1.20091 & 1.26302 & 1.29830 \\
\hline 4 & 0.410731 & 0.67723 & 0.84137 & 0.93487 & 0.98030 & 0.99334 & 0.98480 \\
\hline 5 & 0.391566 & 0.61828 & 0.73726 & 0.78731 & 0.79434 & 0.77533 & 0.74123 \\
\hline 6 & 0.373044 & 0.56325 & 0.64397 & 0.66050 & 0.64096 & 0.60249 & 0.55537 \\
\hline 7 & 0.355320 & 0.51258 & 0.56147 & 0.55285 & 0.51585 & 0.46687 & 0.41489 \\
\hline 8 & 0.338413 & 0.46623 & 0.48903 & 0.46208 & 0.41444 & 0.36109 & 0.30931 \\
\hline 9 & 0.322302 & 0.42397 & 0.42567 & 0.38584 & 0.33258 & 0.27890 & 0.23027 \\
\hline 10 & 0.306956 & 0.38548 & 0.37038 & 0.32198 & 0.26666 & 0.21521 & 0.17124 \\
\hline
\end{tabular}


Table 3. Variances of record statistics for $\alpha=5$.

\begin{tabular}{|c|c|c|c|c|c|c|c|}
\hline$n$ & $\beta=0.5$ & $\beta=1.0$ & $\beta=1.5$ & $\beta=2.0$ & $\beta=2.5$ & $\beta=3.0$ & $\beta=3.5$ \\
\hline 1 & 0.167379 & 0.18670 & 0.24257 & 0.50531 & 0.75025 & 0.96801 & 1.15716 \\
\hline 2 & 0.164047 & 0.15148 & 0.17206 & 0.32023 & 0.41989 & 0.47905 & 0.50834 \\
\hline 3 & 0.144385 & 0.12165 & 0.09685 & 0.16464 & 0.19130 & 0.19283 & 0.18112 \\
\hline 4 & 0.123282 & 0.11363 & 0.04886 & 0.07689 & 0.07888 & 0.06969 & 0.05732 \\
\hline 5 & 0.104164 & 0.10124 & 0.02216 & 0.03309 & 0.02972 & 0.02262 & 0.01586 \\
\hline 6 & 0.087666 & 0.01970 & 0.00847 & 0.01281 & 0.00989 & 0.00619 & 0.00342 \\
\hline 7 & 0.073668 & 0.01654 & 0.00274 & 0.00406 & 0.00253 & 0.00105 & 0.00019 \\
\hline 8 & 0.061864 & 0.01347 & 0.00072 & 0.00064 & 0.00013 & 0.00025 & 0.00038 \\
\hline 9 & 0.051935 & 0.01075 & 0.00162 & 0.00049 & 0.00045 & 0.00041 & 0.00032 \\
\hline 10 & 0.043591 & 0.00844 & 0.00170 & 0.00071 & 0.00046 & 0.00031 & 0.00019 \\
\hline \multicolumn{8}{|c|}{ Table 4. Variances of record statistics for $\alpha=10$} \\
\hline$n$ & $\beta=0.5$ & $\beta=1.0$ & $\beta=1.5$ & $\beta=2.0$ & $\beta=2.5$ & $\beta=3.0$ & $\beta=3.5$ \\
\hline 1 & 0.202642 & 0.21372 & 0.42774 & 0.99905 & 1.65322 & 2.35327 & 3.07455 \\
\hline 2 & 0.200805 & 0.21869 & 0.36211 & 0.78764 & 1.20893 & 1.59646 & 1.93754 \\
\hline 3 & 0.187063 & 0.16113 & 0.27761 & 0.56422 & 0.80391 & 0.98518 & 1.11085 \\
\hline 4 & 0.171651 & 0.15407 & 0.20626 & 0.39123 & 0.51685 & 0.58719 & 0.61451 \\
\hline 5 & 0.156774 & 0.14835 & 0.15098 & 0.26688 & 0.32645 & 0.34338 & 0.33308 \\
\hline 6 & 0.142960 & 0.15129 & 0.16955 & 0.18022 & 0.20381 & 0.19824 & 0.17791 \\
\hline 7 & 0.130290 & 0.14351 & 0.10792 & 0.12082 & 0.12613 & 0.11321 & 0.09386 \\
\hline 8 & 0.118719 & 0.14119 & 0.05667 & 0.08052 & 0.07748 & 0.06405 & 0.04893 \\
\hline 9 & 0.108166 & 0.10988 & 0.04045 & 0.05338 & 0.04726 & 0.03591 & 0.02519 \\
\hline 10 & 0.098548 & 0.09673 & 0.02873 & 0.03521 & 0.02863 & 0.01993 & 0.01279 \\
\hline
\end{tabular}

\section{Concluding remarks}

In this article, we provide explicit expressions for the quantiles, moments, moment generating function, conditional moments, hazard rate, mean residual lifetime, mean past lifetime, mean deviation about mean and median, the stochastic or- dering, various entropies, stress-strength parameter, Bonferroni and Lorenz curve and order statistics. The explicit expressions as well as several recurrence rela- tions for the single and product moments of record values from Burr XII $(\alpha$, $\beta)$ model are established. We also computed the means and variances of the upper record values.

A future work may be to derive estimation procedures for the $\operatorname{Burr} \operatorname{XII}(\alpha, \beta)$ distribution based on order statistics, generalized order statistics and dual gen- eralized order statistics. Another future work may be to characterize the $\operatorname{Burr} \operatorname{XII}(\alpha, \beta)$ distribution based on order statistics, generalized order statistics and dual generalized order statistics.

\section{Acknowledgment}

The author would like to thanks two unknown referees and the editors for many valuable suggestions which have helped to improve the paper significantly. 


\section{References}

[1] Abdel-Hamid, A. H. (2009): Constant-Partially accelerated life tests for Burr XII distribution with progressive type II censoring, Computational Statist., 53, 2511-2523.

[2] Abdel-Ghaly, A. A., Al-Dayian, G. R. and Al-Kashkari, F. H. (1997). The use of Burr type XII distribution on software reliability growth modeling, Microelectronics and Reliability, 37, 305-313.

[3] Ahsanullah, M. (1995). Record Statistics. Nova Science Publishers, New York.

[4] AL-Hussaini, E. K. (2003). Bayesian predictive density of order statistics based on finite mixture models, Journal of Statistical Planning and Inference, 113, 15-24

[5] AL-Hussaini, E. K. and Ahmad, A. A. (2003). On bayesian interval prediction of future records, Test, 12, 79-99.

[6] Arnold, B.C., Balakrishnan, N. and Nagaraja, H.N. (1992). A first course in order. John Wiley and Sons, New York.

[7] Arnold, B.C., Balakrishnan, N. and Nagaraja, H.N. (1998). Record, John Wiley and Sons, New York.

[8] Balakrishnan, N. and Ahsanullah, M. (1993). Relations for single and product moments of record values from exponential distribution, J. Appl. Statist. Sci., 2, 73-87.

[9] Balakrishnan, N. and Ahsanullah, M. (1994). Recurrence relations for single and product moments of record values from generalized Pareto distribution. Comm. Statist. Theory Methods, 23, 2841-2852.

[10] Balakrishnan, N. and Cohen, A. C. (1991). Order statistics and inference: esti- mation methods. Boston, MA:Academic Press.

[11] Bonferroni C.E. (1930). Elmenti di statistica generale. Libreria Seber, Firenze.

[12] Burr, I. W., (1942). Cumulative frequency functions, Ann. Math. Statist., 13, 215-232.

[13]Burr I. W. and Cislak, P. J. (1968). On a general system of distributions: I. Its curve shaped characteristics; II. The sample median, J. Amer. Statist. Assoc, 63 627-635.

[14] Chandler, K. N. (1952). The distribution and frequency of record values. J. Roy. Statist. Soc., Ser B, 14, 220-228. 
[15] Grunzien, Z. and Szynal, D. (1997). Characterization of uniform and exponen- tial distributions via moments of the kth record values with random indices, Appl. Statist. Sci., $5,259-266$.

[16] Glick, N. (1978). Breaking records and breaking boards, Amer. Math. Monthly, $85,2-26$.

[17] Kenney, J. F. and Keeping, E. (1962). Mathematics of Statistics. D. Van Nostrand Company.

[18] Kumar, D. (2015). Explicit expressions and statistical inference of generalized Rayleigh distribution based on lower record values, Mathematical Methods of Statistics, 24, 225241.

[19]Kumar, D. Jain, N. and Gupta, S. (2015). The type I generalized half logis- tic distribution based on upper record values, Journal of Probability and Statistics, 2015 , 01-11.

[20]Kumar, D. (2016). kth lower record values from of Dagum distribution, Discus- siones Mathematicae Probability and Statistics, 36, 25-41.

[21]Kumar, D. (2016). Ratio and inverse moments of Marshall-Olkin extended Burr type XII distribution based on lower generalized order statistics, Journal of Data Science, 14, 53-66.

[22]Kumar, D., Kumar, M., Saran, J. and Jain, N. (2017). The Kumaraswamy- Burr type III distribution based on upper record values, American Journal of Mathematical and Management Sciences, (To appear).

[23]Lin, G.D., (1986). On moment problem, Tohoku Math. Journal, 38, 595-598.

[24]Li, X, Shi, Y., Wei, J. and Chai, J. (2007). Empirical Bayes estimators of reliability performances using LINEX loss under progressively type-II censored

[25] Moors, J. J. A. (1988). A quantile alternative for kurtosis. Journal of the Royal Statistical Society. Series D (The Statistician), 37, 25-32.

[26] Moore, D. and Papadopoulos, A. S. (2000). The Burr type XII distribution as a failure model under various loss functions, Microelectronics and Reliability, 40, 2117-2122.

[27] Mousa, M. A. M. A. and Jaheen, Z. F. (2002). Statistical inference for the Burr model based on progressively censored data, Computers \& Mathematics with Applications, 43, 14411449.

[28]Nevzorov, V. B. (1987). Records. Theo. Prob. Appl., 32, 201-228.

[29]Pawlas, P. and Szynal, D. (1998). Relations for single and product moments of kth record values from exponential and Gumbel distributions, J. Appl. Statist. Sci., 7, 53-61.

[30] Pawlas, P. and Szynal, D. (1999). Recurrence relations for single and product moments of kth record values from Pareto, generalized Pareto and Burr distributions, Comm. Statist. Theory Methods, 28, 1699-1709.

[31]Pawlas, P. and Szynal, D. (2000). Recurrence relations for single and product moments of kth record values from Weibull distribution and a characteri-zation, J. Appl. Stats. Sci., $10,17-25$.

[32]Resnick, S.I., (1973). Extreme values, regular variation and point processes, Springer-Verlag, New York. 
[33]R'enyi, A. (1961). On measures of entropy and information. In: Proceedings of the 4th Berkeley Symposium on Mathematical Statistics and Probability, I, 547-561. University of California Press, Berkeley

[34] Singh, B. P. and Shukla, U. (2017). An Extension of Poisson Distribution and its Applications in Human Reproduction, Journal of Data Science, 15, 155- 166.

[35] Shaked M, Shanthikumar J. G. (1994). Stochastic orders and their applications, Boston, MA: Academic Press.

[36] Shorrock, R. W. (1973). Record values and inter-record times, J. Appl. Probab., $10,543-555$.

[37] Song, K. S. (2001). R'enyi information, loglikelihood and an intrinsic distribution measure. J. Statist. Plann. Inference, 93, 51-69.

[38] Sultan, K. S. (2007). Record values from the modified Weibull distribution and applications, International Mathematical Forum, 41, 2045-2054.

[39]Wang, F. K. and Keats, J. B. (1996). Maximum likelihood estimation of the Burr type XII parameters with censored and uncensored data, Microelectronics and Reliability, 36, 359362.

[40]Wu, J. W. and Yu, H. Y. (2005). Statistical inference about the shape parameter of the Burr type XII distribution under the failure-censored sampling plan, Applied Mathematics and Computation, 163, 443-482.

[41]Wu, S. J., Chen, Y. J. and Chang, C. T. (2007). Statistical inference based on progressively censored samples with random removals from the Burr type XII distribution, Journal of Statistical Computation and Simulations, 77, 19-27.

[420Zimmer, W. J., Keats, J. B. and Wang, F. K. (1998). The Burr XII distribution in reliability analysis, Journal of Quality Technology, 30, 386-394.

Received June 25, 2014; accepted September 28, 2014.

Devendra Kumar Department

of Statistics

Central University of Haryana

Mahendergarh, India

Email: devendrastats@gmail.com 
